\title{
APPLYING UNCERTAINTY REDUCTION STRATEGY FOR IMPROVING PERFORMANCE OF QUESTIONNAIRE TECHNIQUE OF SOLVING COLD USER PROBLEM
}

\author{
Abubakar, M. ${ }^{1}$ and Umar, $K^{2}$ \\ ${ }^{1}$ Department of Computer Science, Faculty of Computer Science and Information Technology \\ Bayero University, Kano-Nigeria. \\ ${ }^{2}$ Department of Software Engineering, Faculty of Computer Science and Information Technology \\ Bayero University, Kano-Nigeria. \\ *Correspondence author: muhammadabubakar08155@gmail.com
}

\begin{abstract}
Product recommendation systems are information filtering systems that uses ratings and predictions to make new product suggestions. There are many product recommendation system techniques in existence, these include collaborative filtering, content based filtering, knowledge based filtering, utility based filtering and demographic based filtering. Collaborative filtering techniques is known to be the most popular product recommendation system technique. Itutilizesuser'sprevious productratings to make new product suggestions. However collaborative filtering have some weaknesses, which include cold start, grey sheep issue, synonyms issue. However the major weakness of collaborative filtering approaches is cold user problem. Cold user problem is the failure of product recommendation systems to make product suggestions for new users. Literature investigation had shown that cold user problem could be effectively addressed using active learning technique of administering personalized questionnaire. Unfortunately, the result of personalized questionnaire technique could contain some user preference uncertainties where the product database is too large (as in Amazon). This research work addresses the weakness of personalized questionnaire technique by applying uncertainty reduction strategy to improve the result obtained from administering personalized questionnaire.In our experimental design we perform fourdifferent experiments; Personalized questionnaire approach of solving user based coldstart was implemented using Movielens dataset of $1 \mathrm{M}$ size, Personalized questionnaire approach of solving user based cold start was implemented using Movielens dataset of $10 \mathrm{M}$ size,Personalized questionnaire with uncertainty reduction was implemented using Movielens dataset of $1 \mathrm{M}$ size, and also Personalized questionnaire with uncertainty reduction was implemented using Movielens dataset of $10 \mathrm{M}$ size. The experimental result shows RMSE, Precision and Recall improvement of $0.21,0.17$ and 0.18 respectively in $1 M$ dataset and $0.17,0.14$ and 0.20 in $10 \mathrm{M}$ dataset respectively over personalized
\end{abstract} questionnaire.

\section{INTRODUCTION}

The rise of e-commerce websites lead to the growth of the online product recommender systems, and sincemid-1990's product recommender system have become a significant study domain (Adovicius et al., 2005).Product recommender systems are one of the deeprooted artificial intelligence application in contemporary computer science (Sadeh,2002).Areas in which recommender systems are applicable include business, videos, games, You tube, Google, facebook, Netflix, Amazon, Movielens and so on. Taysuzoglu (2018) defined product recommender system as an information filtering technique that uses ratings and predictions to make new recommendations. Okaka (2018) defined product recommender system as a branch of information filtering system that predict the rating that a user would give to an item. There are various techniques proposed for product recommendation systems in existence, among which arecollaborative filtering technique, content based filtering, utility and knowledge based filtering (Jazeyeriy et al., 2018). Recommendation systems aresignificant tool for many commercialapplications. The intelligence of recommendation systems can be seenin their ability to learn from users preferences, and then recommend product that satisfy their tastes. Pozoet al.(2018) worked on active learning 
BAJOPAS Volume 13 Number 2,December, 2020

method for dealingwithcold start in A new user to a product recommendation system is presented a questionnaire in order to know his preferences. However Result gotten from personalized questionnaires could be too large and might contain some elements of user preference uncertainty. Many questions might challenge the willingness of the user to fill the questionnaire. Mohammad-Hossein (2014) worked on addressing cold start problem by askinga new user to rate an item. The problem with this technique is that new user need to rate many items before items of preference will be recommended to him.Zhu(2018) worked on addressing item based cold start byusing attribute-driven active learning technique. Patilet al.(2018) worked on demographic collaborative and content filtering technique based hybrid recommendation system. Vishwajithet al.(2019) worked on hybrid recommender system for therapy recommendation. The collaborative filtering idea is transformed to the therapy recommendation domain. The researchers considering therapies as items and therapy response as a user's preference. Han(2019) worked on hybrid recommender system for patient doctor matching in primary health care. The researcher developed a hybrid recommendation system by learning latent representations for patients and doctors from their interactions and meta data. Neysianiet al.(2019) worked on improving performance of association rule based collaborative filtering recommendation systems using genetic.Collaborative filtering is known to be the first recommendation system technique (Adovicius et al., 2005)and also the most used product recommendation system technique (Taysuzoglu,2018).A personalized questionnaire method is a method that engage users to interact with the system by presenting them with a questionnaire to understand their preferences. On the other hand, Passive learning uses random existing users rating, which causes learning user preference to be slow. Active learning is part of machine learning. Machine learning is a research area which focuses on design and development of novel algorithms, which are used in solving a large variety of tasks such as regression and classification task.However Collaborative filtering weaknesses include cold start, scalability, synonyms issue, data scarcity, e.t.c. Literature investigation had shown that the dominantissue of collaborative filtering is cold user problem(Pozo, 2018); (Mohammad-Hossein, 2014); (Karimi, 2011)\& (Kohrs\&Merialdo, 2001). It is the failure of product recommendation system to make recommendation to a new user. A new user that recommender systems.

appropriate recommendations are not recommended to him/her may restrain from the product recommendation system. Researchers addressed cold user problem using active learning by asking the user some questions before matching him to the cluster of users having the same interest as the new user, so that similar recommendations will be made. However results of questionnaire could be too large and could contain more elements of user preference uncertainties. In this research,a personalized questionnaire is presented to the new user. Highly rated items from the result of personalized questionnaire are presented for to the new user to rate based on his/her preferences prior to user cluster assignment. This has the tendency of reducing uncertainty inherent in the result of personalized questionnaire. Four experiments were conducted. Personalized questionnaire approach of solving user coldstart implemented using Movielens dataset of $1 \mathrm{M}$ size. Personalized questionnaire approach of solving user cold start implemented using Movielens dataset of $10 \mathrm{M}$ size. Personalized questionnaire with uncertainty reduction implemented using Movielens dataset of $1 \mathrm{M}$ size.Personalized questionnaire with uncertainty reduction implemented using Movielens dataset of $10 \mathrm{M}$ size.The experimental result shows RMSE, Precision and Recall improvement of $0.21,0.17$ and 0.18 respectively in $1 \mathrm{M}$ dataset and $0.17,0.14$ and 0.20 in $10 \mathrm{M}$ dataset respectively over personalized questionnaire.

\section{MATERIALS AND METHODS Principles}

Active learning techniques were used in order to achieve user preference. At first, existing users having similar interest were clustered. A cold user is asked the genre(s) of his interest. Items are presented to the user based on the genre(s) provided by the cold user. Highly rated items from the ones chosen by the cold user are presented for the cold user to rate. Requesting a genre(s) and selecting some items of interest followed by rating highly rated items will be of help towards satisfying user preferences.

\section{Architecture}

In the proposed system architecture we have three modules, which are the clustering module, interest acquirement module and user cluster assignment module. The interestacquirement module has two components, which are Personalized Questionnaire Analysis and Uncertainty Reduction. Figure 1 contains the architectural design of the proposed system. 


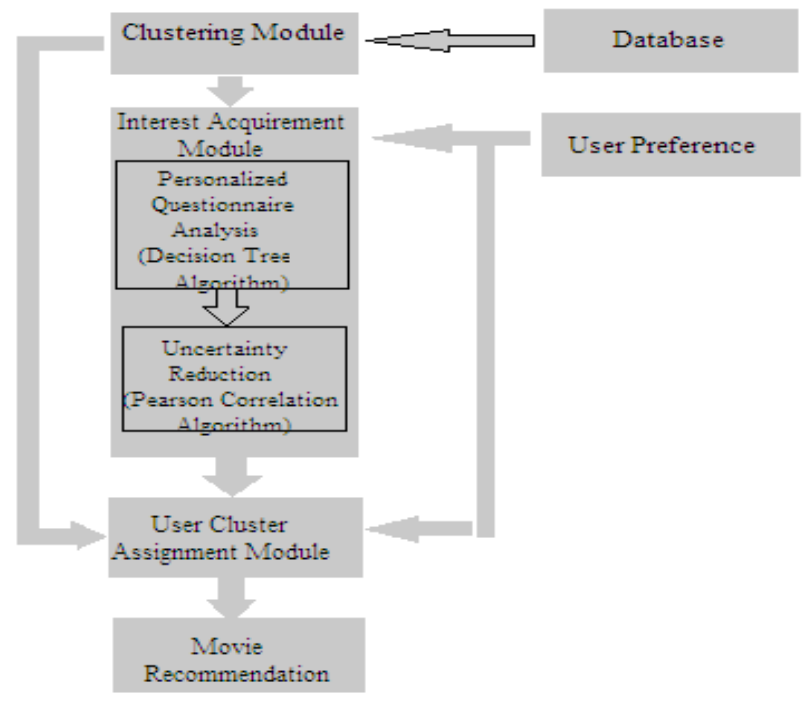

Figure 1: Architectural Design of the proposed system.

\section{Clustering module}

In this module, clusters were formed. Users having similar interest are grouped into various clusters. Kmeans clustering technique will be used. The benefit of clustering is to know the users having the same interest as the cold user.

\section{Interest Acquirement Module}

In this module we have two components, the personalized questionnaire component and uncertainty reduction component, new user is presented a personalized questionnaire for him to answer. Decision tree algorithm is used for implementation. Uncertainty reduction technique is applied to the result of the personalized questionnaire. The new user is presented highly rated item from the result of the personalized questionnaire for him/her to rate prior to user cluster assignment. Pearson correlation algorithm is used for implementation. Figure 2 contains the decision tree diagram of the personalized questionnaire.

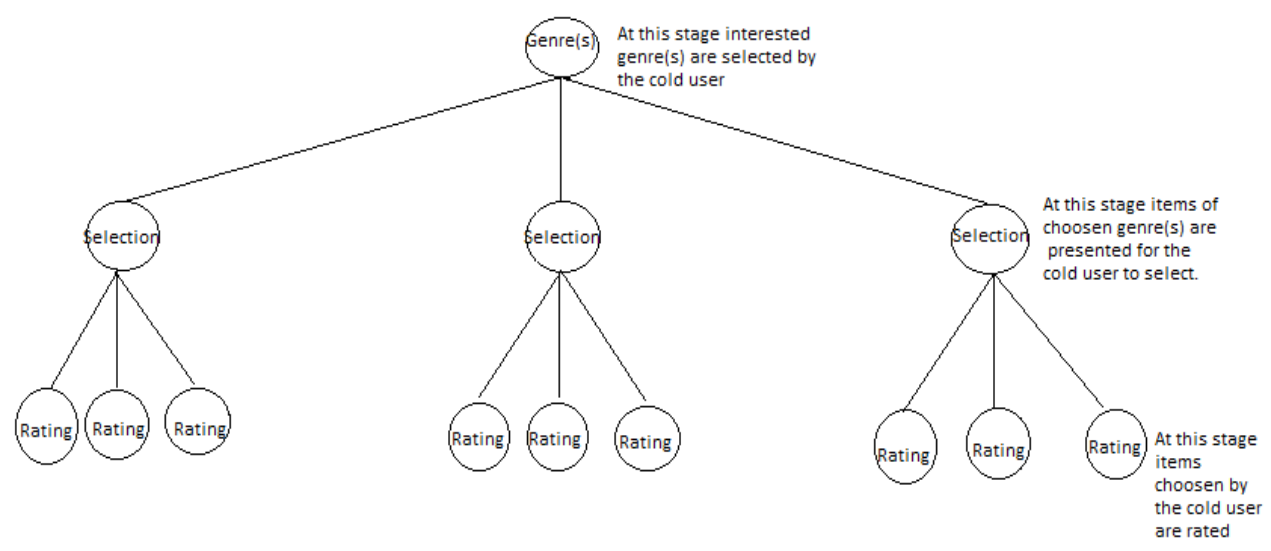

Figure 2: Decision tree structure of the personalized questionnaire technique.

\section{User Cluster Assignment Module}

In this module, users having similar interest are assigned to the same cluster. Similar recommendations are made to people in the same cluster.

\section{Algorithm}

Active learning technique are usually implemented using decision tree. The basic steps in decision tree algorithm are presented in algorithm1. The decision tree has three child nodes. The decision three is followed by the basic steps of Pearson correlation algorithm. The decision three is used in presenting personalized questionnaire. Highly rated items from the result of personalized questionnaire are presented to the new user to rate. The highly rated items are presented using Pearson correlation algorithm. 
Algorithm 1: Basic Decision tree Algorithm improved by Pearson correlation Algorithm.

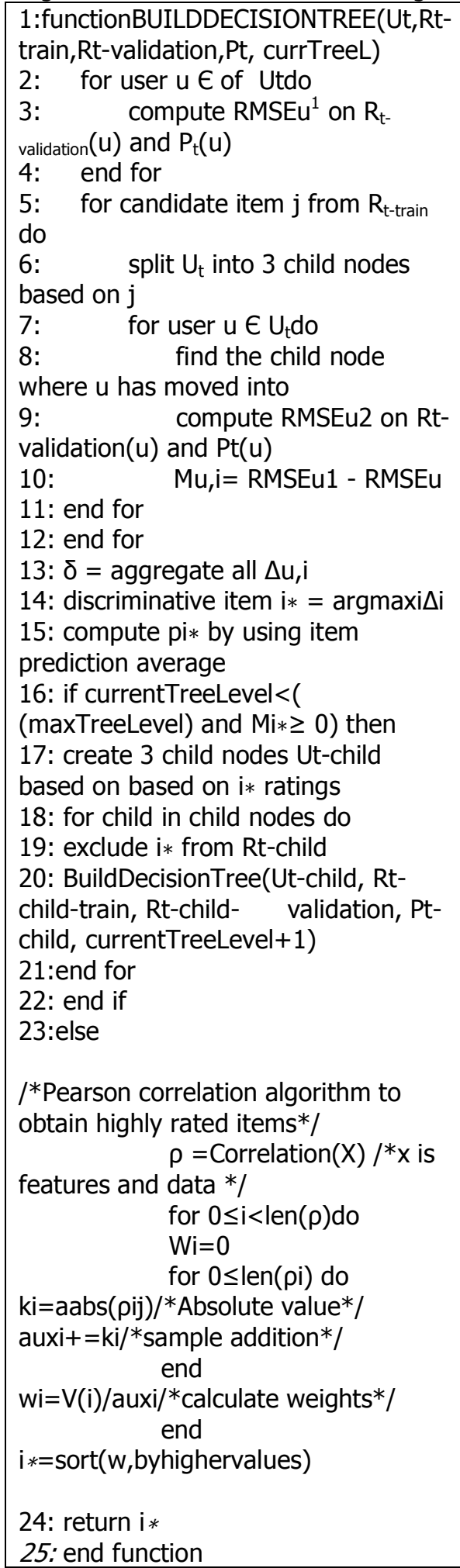

\section{RESULTS}

In the section below, result of the personalized questionnaire with uncertainty reduction were presented. It was obtained that the result of personalized questionnaire approach of solving cold user problem with uncertainty reduction was better than that of personalized questionnaire approach alone. Below in Table 2 is the result of personalized questionnaire with uncertainty reduction technique. 
BAJOPAS Volume 13 Number 2,December, 2020

Table 2:Result of previous and proposed technique

\begin{tabular}{llcccc}
\hline DATA SIZE & \multicolumn{1}{c}{ MODEL } & RMSE & PRECISION & \multicolumn{2}{c}{ RECALL } \\
\hline $1 \mathrm{M}$ & $\begin{array}{l}\text { PREVIOUS MODEL } \\
\text { (P.Q. Model) } \\
\text { PROPOSED MODEL }\end{array}$ & 0.698 & 0.583 & 0.636 & \\
& $\begin{array}{l}\text { (P.Q. + U.R) } \\
\text { PREVIOUS MODEL } \\
\text { (P.Q. Model) } \\
\text { PROPOSED MODEL } \\
\text { (P.Q. + U.R Model) }\end{array}$ & 0.488 & 0.750 & 0.818 & 0.439 \\
& & 0.866 & 0.486 & 0.639 \\
\hline
\end{tabular}

Across all the evaluations, in Table 2, results show that the personalized questionnaire technique of solving cold user problem with uncertainty reduction approach achieve better RMSE, precision and recall in both $1 \mathrm{M}$ and $10 \mathrm{M}$ size than the work of pozo(2018). The proposed strategy was implemented and the experimental result shows RMSE, Precision and Recall improvement of $0.21,0.17$ and 0.18 respectively in $1 \mathrm{M}$ dataset and $0.17,0.14$ and 0.20 in $10 \mathrm{M}$ dataset respectively over personalized questionnaire as shown in Table 2, that best results were achieved where we have small dataset. From the result it can be seen that personalized questionnaire technique with uncertainty reduction technique have better results.

\section{CONCUSION}

In this research, active learning technique of uncertainty reduction was applied to personalized questionnaire approach of solving cold user problem. We carried out for experiments. Personalized questionnaire approach of solving user coldstart implemented using Movielens dataset of $1 \mathrm{M}$ size. Personalized questionnaire implemented using Movielens dataset of $10 \mathrm{M}$ size. Personalized questionnaire with uncertainty reduction implemented using

\section{REFERENCES}

Adovicius, T. (2005). "A hidden Markov model for collaborative filtering." Management Information Systems Quarterly, Forthcoming.

Alhijawi, B. J. M. (2017). The Use of the Genetic Algorithms in the Recommender Systems (Doctoral dissertation, Hashemite University).

Ayyaz, S., Qamar, U., \& Nawaz, R. (2018). HCFCRS: A Hybrid Content based Fuzzy Conformal Recommender System for Providing recommendations with confidence. PloS one, 13(10), e0204849.

Brigui-Chtioui, I., Caillou,P., \& Negre, E.(2018).Intelligent Digital Learning: Agent-Based Recommender System.

Divya, N., Sandhiya, S., Liz, D. A. S., \&GNANAOLI, P. (2018). A collaborative filtering based recommender system usingrating prediction.International Journal of Pure and Applied Mathematics, 119(10), 1-7.

Drdák, J. (2018). Optimalizacedoporučovacíchalgoritmů. Jazayeriy, H., Mohammadi, S., \& Shamshirb and, S. (2018). Afast recommender system for cold user using
Movielens dataset of $1 \mathrm{M}$ size.Personalized questionnaire with uncertainty reduction implemented using Movielens dataset of 10M size.We found improvement in terms of RMSE, recall and precision. The proposed strategy was implemented and the experimental result shows RMSE, Precision and Recall improvement of $0.21,0.17$ and 0.18 respectively in $1 \mathrm{M}$ dataset and $0.17,0.14$ and 0.20 in $10 \mathrm{M}$ dataset respectively over personalized questionnaire.

categorized items. Mathematical and Computational Applications, 23(1), 1.

Eksombatchai, C., Jindal, P., Liu, J. Z., Liu, Y., Sharma, R., Sugnet, C., ...\& Leskovec, J. (2018, April). Pixie: A system for recommending $3+$ billion items to $200+$ million users in real-time. In Proceedings of the 2018 World Wide Web Conference (pp. 1775-1784). International World Wide Web Conferences Steering Committee. Ekstrand, M. D., Tian, M., Azpiazu, I. M., Ekstrand,J. D., Anuyah, O., McNeill, D., \& Pera, M. S. (2018, January). All the cool kids, how do they fit in?: Popularity and demographicbiases in recommender evaluation and effectiveness. In Conference on Fairness, Accountability and Transparency (pp. 172-186).

Geetha, G., Safa, M., Fancy, C., \&Saranya, D. (2018, April). A hybrid approach usingcollaborative filtering and content based filtering for recommender system. In Journal of Physics: Conference Series (Vol. 1000, No. 1, p. 012101). IOP Publishing.

Gohari, F. S., \&Tarokh, M. J. (2016). A New Hybrid Collaborative Recommender 
BAJOPAS Volume 13 Number 2,December, 2020 Using Semantic Web Technology and Demographic data. International Journal of Information and Communication Technology Research, 8(2), 51-61.

Gunaruwan, T. Lalithasiri, and W. Neluka Gunasekara. (2007)." A Modular Framework for Extensible and AdaptableRecommendation Algorithms." NSBMJournal of Management 2.1.

Guo, Y., Yin, C., Li, M., Ren, X., \& Liu, P. (2018). Mobile e-commerce recommendation system based on multi-source information fusion for sustainable ebusiness. Sustainability, 10(1), 147.

Han, Q., Ji, M., de Troya, I. M. D. R., Gaur, M., \& Zejnilovic, L. (2018, October). A Hybrid commender System for PatientDoctor Matchmaking in Primary Care. In 2018IEEE 5th International Conference on Data Science and Advanced Analytics (DSAA)(pp. 481-490). IEEE.

He, X., He, Z., Du, X., \& Chua, T. S. (2018, June).Adversarial personalized ranking forrecommendation. In The 41st InternationalACM SIGIR Conference on Research \&Development in Information Retrieval (pp. 355-364). ACM.

Jazayeriy, Hamid, Saghi Mohammadi, and Shahaboddin Shamshirband. "A fast recommender system for cold user using categorized items." Mathematical andComputational Applications 23.1 (2018): 1.Uçar, Ahmet, Karl E. Lonngren, and Er-Wei Bai. "Synchronization of the unified chaotic systems via active control." Chaos, Solitons \& Fractals 27.5 (2006): 12921297.

Kangas, S.(2002). "Collaborative filtering and recommendation systems." VTT information technology.

Karimi, R., Freudenthaler, C., Nanopoulos, A., \&Schmidt-Thieme, L. (2011, April). Active learning for aspect model in recommender systems. In 2011 IEEE Symposium on Computational Intelligence and Data Mining (CIDM) (pp. 162-167). IEEE.

Kurihara, Kenichi, Max Welling, and Yee WhyeTeh..(2007) "Collapsed Variational DirichletProcess Mixture Models." IJCAI. Vol. 7.

Mahadi, M. I., Zainuddin, N., Shah, N. B. A., Naziron, N. A., \& Rum, S. F. M. E-Halal Restaurant Recommender System UsingCollaborative Filtering Algorithm.

Merialdo, A. K. B. (2001). Improving collaborative filtering for new-users by smart object selection.
Nadimi-Shahraki, M. H., \& Bahadorpour, M. (2014).Cold-start problem in collaborativerecommender systems: efficient methods based on ask-to-rate technique. Journal of computingand information technology, 22(2), 105-113.

Nouh, R. M., Lee, H. H., Lee, W. J., \& Lee, J. D.(2019). A Smart Recommender Based on Hybrid Learning Methods for Personal WellBeing Services. Sensors, 19(2), 431.

Neysiani,B.,Soltani,N., Mofidi, R., \& NadimiShahraki,M.(2019). Improving Performance of Association RuleBasedCollaborative Filtering Recommendation Systems using Genetic Algorithm, 02(06).

Patil, P., Sata, M., Fancy, C. and Saranya, D. (2018).Demographic collaborative and content filtering technique based hybrid recommender system, 10(2-6).

Pazzani, M. J. (1999). A framework for collaborative, content-based and demographic filtering.Artificial intelligencereview, 13(5-6), 393-408.

Pozo, M., Chiky, R., Meziane, F., \& Métais, E.(2018, September). Exploiting past users' interests and predictions in an active learning method for dealing with cold start in recommender systems. In Informatics (Vol. 5, No. 3, p. 35). Multidisciplinary DigitalPublishing Institute.

Russel, Stuart, and Peter Norvig. (2010)."Artificial Intelligence: A Modern Approach Third Edition." Person Education, Boston Munich

Smith, J., Weeks, D., Jacob, M., Freeman, J., \&Magerko, B. (2019, March). Towards a Hybrid Recommendation System for a Sound Library. In IUI Workshops.

Sun, M., Li, F., \& Zhang, J. (2018). A multimodality deep network for cold-start recommendation. Big Data and Cognitive Computing, 2(1), 7.

Taysuzoglu,Y. (2018) "Collaborative filteringenhanced by demographic data", MemorialUniversity of Newfoundland".

Vishwajith, V., Kaviraj, S., \& Vasanth, R.(2019).Hybrid Recommender System for Therapy Recommendation, 13(5-6), 393-408.

Vozalis, Manolis, and Konstantinos G. Margaritis."Collaborative filtering enhanced bydemographic correlation." AIAI symposium on professional practice in AI, of the 18th world computer congress. 2004. 
BAJOPAS Volume 13 Number 2,December, 2020

Wang, Y., Wang, M., \& Xu, W. (2018). A sentiment-enhanced recommender system recommendation: A analyticsframework. hybrid
formovie Communications andMobileComputing, 2018.
Zarzour, H., Al-Sharif, Z., Al-Ayyoub, M.,\&Jararweh, Y. (2018, April). A new collaborativefiltering recommendation algorithm based ondimensionality reduction and clusteringtechniques. In 2018 9th International Conference on Information and CommunicationSystems (ICICS) (pp. 102-106). IEEE.

Zhu, Y., Lin, J., He, S., Wang, B., Guan, Z., Liu, H., \& Cai, D. (2019). Addressing the item cold-start problem by attributedriven active learning. IEEE Transactions on Knowledge andData Engineering. 